\title{
Diferenciais intra-urbanos de mortalidade em Belo Horizonte, Minas Gerais, Brasil, 1994: revisitando o debate sobre transições demográfica e epidemiológica
}

\author{
Intra-urban mortality differentials in Belo \\ Horizonte, Minas Gerais State, Brazil, 1994: \\ revisiting the debate on demographic and \\ epidemiological transitions
}

Rômulo Paes-Sousa 1,2

\footnotetext{
1 Centro Nacional

de Epidemiologia.

Setor de Autarquias Sul,

Quadra 4, Bloco N, 6o Andar,

Sala 604, Brasília, DF

70070-040, Brasil.

romulo.sousa@funasa.gov.br

2 Pós-Graduação em

Ciências Sociais, Pontifícia

Universidade Católica

de Minas Gerais.

Av. Dom Gaspar 500,

Prédio 40, 2o andar,

Belo Horizonte, $M G$

30535-610, Brasil.
}

\begin{abstract}
Demographic and epidemiological transition models have seldom been debated within the Brazilian scientific community. This study aims to critically review the studies based on such transition models, analyzing their contributions and limitations for health research in Brazilian cities. Data from the city of Belo Horizonte were used to illustrate the theoretical issues raised in this paper. A total of 10,558 death certificates from 1994 were classified according to underlying cause of death and place of residence (75 geographical areas). Areas were classified according to the proportion of heads of households with low formal education. Population age structures and age- and sex-adjusted mortality rates in the areas were compared. Mortality rates indicate that Belo Horizonte is experiencing a multiple and unequal epidemiological transition process. In the poorer areas, infectious diseases have been replaced by homicides as cause of death in adulthood. The findings suggest that large Brazilian cities have mortality patterns that vary according to social and economic differentials.
\end{abstract}

Key words Inequality; Epidemiological Transition; Demographic Transition; Urbanisation

Resumo Modelos de transições demográfica e epidemiológica não têm sido debatidos com grande freqüência pela comunidade acadêmica brasileira. Este estudo tem por objetivo rever criticamente os estudos referentes a estes modelos de transição, analisando suas contribuições e limites à investigação em saúde das populações urbanas do Brasil. Dados do Município de Belo Horizonte são usados para ilustrar os aspectos teóricos levantados neste artigo. Um total de 10.558 declarações de óbito do ano de 1994 foram processadas visando à classificação da causa básica do óbito e local de residência - 75 unidades geográficas. As áreas foram classificadas de acordo com a proporção de chefes de domicílio com baixa escolaridade. Estruturas populacionais e taxas de mortalidade ajustadas por sexo e idade das áreas foram comparadas. As taxas de mortalidade indicam que Belo Horizonte está experimentando múltiplos e desiguais processos de transição epidemiológica. Nas áreas mais pobres, as doenças infecciosas dos adultos têm sido substituídas pelos homicídos. Em geral, os achados sugerem que as grandes cidades brasileiras possuem padrões de mortalidade que variam de acordo com os diferenciais sociais e econômicos.

Palavras-chave Desigualdade; Transição Epidemiológica; Transição Demográfica 


\section{Introdução}

O rápido e intenso processo de urbanização do Brasil tem colocado dificuldades adicionais ao sistema de saúde do país. Se, por um lado, essa condição implica maior acesso aos bens e serviços ligados à saúde das populações; por outro, implica submissão de amplos contingentes humanos à carga maior de riscos físicos (poluição) e sócio-ambientais (hiper-adensamento populacional e violências). As cidades passam a vivenciar um perfil epidemiológico mais complexo, demandando maior gasto e maior complexidade no atendimento dessas demandas. Nesse sentido, a teoria da transição epidemiológica pode ajudar na compreensão do padrão epidemiológico emergente das áreas urbanas brasileiras.

Este texto enfoca o debate sobre as transições demográfica e epidemiológica, utilizando dados referentes ao Município de Belo Horizonte, Minas Gerais, como exemplificação, na discussão dos aspectos teóricos levantados ao longo do texto. Na seção final deste trabalho, são analisadas as implicações do uso de modelos de transição demográfica e epidemiológica nas políticas de saúde.

\section{Transição demográfica}

A transição demográfica é definida como processo de modernização global com base em determinado padrão demográfico "tradicional”, até sua conversão em um padrão demográfico moderno (Chesnais, 1992). O padrão tradicional é caracterizado por altos níveis de mortalidade e de fecundidade e o moderno caracteriza-se por baixos níveis em relação aos dois elementos da dinâmica populacional citados. Chesnais (1992) define três paradigmas para este processo: (a) o princípio da anterioridade do declínio da mortalidade sobre a queda da natalidade; (b) o modelo da transição reprodutiva em duas fases, declínio da nupcialidade seguido da limitação da natalidade; (c) a influência do processo de "modernização" no declínio da fertilidade.

Apesar de a ênfase inicial ser dada ao declínio da mortalidade, a teoria de transição demográfica é, sobretudo, uma teoria da natalidade, considerada uma variável dependente do declínio da mortalidade. Alem disso, o declínio da fecundidade e as variáveis econômicas influenciam-se mutuamente no processo de transição demográfica.

O debate sobre a transição demográfica na América Latina converge para um ponto co- mum: todos os países latino-americanos estão envolvidos em um processo de transição (Patarra, 1994).

Por exemplo, o novo padrão demográfico brasileiro é marcado por progressivos declínios das taxas de fecundidade e mortalidade, alteração da estrutura etária, aumento da proporção de idosos e inversão na distribuição da população de áreas urbanas e rurais.

De 1960 a 2000, a taxa de fecundidade total declinou de 6,2 para 2,3. Essa queda significativa deveu-se, especialmente, à adoção expressiva da esterilização feminina, ao uso de contraceptivos orais e, possivelmente, à prática de abortos ilegais.

Em 1996, 75\% das mulheres e homens unidos usavam algum método anticoncepcional. Dentre as mulheres, $40 \%$ haviam sido esterilizadas e $21 \%$ utilizavam contraceptivos orais (BEMFAM, 1997). Não há estatísticas oficiais sobre as práticas abortivas no país, mas é de se esperar que esses métodos tenham tido importante papel no declínio da fecundidade.

Se, por um lado, as mudanças no padrão de fecundidade têm empurrado o Brasil para um modelo de transição avançada, por outro, a expectativa de vida ao nascer é ainda contida pelos altos níveis de mortalidade. Em 1999, a Fundação Instituto Brasileiro de Geografia e Estatística (IBGE, 2001) registrou índices declinantes, mas ainda altos, de mortalidade infantil: 34,8 por mil nascidos vivos. A disparidade nessas medidas vitais sugerem que os progressos nas atitudes contraceptivas não encontram contrapartida eficaz nas políticas de redução da mortalidade, a ponto de alinhar o Brasil a países como Chile e Cuba.

Efetivamente, o declínio da fecundidade parece ter-se consolidado no Brasil. Martine (1996) atribui este processo: ao crescimento da sociedade de consumo, ao crescimento da seguridade social, ao desenvolvimento dos meios de comunicação de massa e aos altos níveis de medicalização da sociedade. Em conjunto, esses fenômenos acabaram por forjar um novo tipo ideal de família que passou a ser aspirado por largos segmentos sociais, vindo a interferir no comportamento reprodutivo de toda a população.

Largos contingentes populacionais deslocaram-se para o espaço urbano após a década de 40. Em 2000, 81,2\% da população brasileira estavam concentrados nas cidades. Contudo, desde os anos 80, tem ocorrido uma desaceleração no processo de "metropolização", isto é, migração maciça em direção às grandes cidades, resultando em uma melhor distribuição da população em cidades de diferentes tamanhos, 
com exceção das capitais dos estados da Amazônia (Martine, 1993, 1994).

O Brasil está completando o seu processo de urbanização. O que significa dizer que a mobilidade no sentido campo-cidade diminuirá de intensidade enquanto a mobilidade no sentido cidade-cidade tenderá a aumentar (Skeldon, 1990)

O processo de urbanização está associado diretamente a mudanças nos padrões de fecundidade e mortalidade. No primeiro caso, pela maior difusão nos centros urbanos dos métodos de contracepção e subseqüente assimilação, pela população de migrantes, de uma nova pauta de valores sócio-culturais. No segundo, a extensão de cobertura dos serviços de saúde, ao final dos anos 80 , promoveu uma redução nas mortes causadas por doenças infecto-contagiosas.

\section{Transição epidemiológica}

O estudo da evolução dos padrões de mortalidade, de forma destacada dos outros elementos da dinâmica populacional, é abordado na teoria da transição epidemiológica.

Nos últimos 30 anos, o declínio da mortalidade e os padrões de mortalidade apresentados em vários países têm sido analisados por intermédio da teoria da transição epidemiológica. Phillips (1994), em uma definição pragmática, considerou esta teoria como um meio de obter-se uma idéia geral dos maiores determinantes da mortalidade e suas interligações com as mudanças demográficas.

$\mathrm{O}$ aspecto mais permanente desta formulação concentra-se no postulação de que as mudanças no padrão de mortalidade ocorreram às custas da progressiva substituição de doenças infecciosas por doenças crônico-degenerativas e causas externas como principais causas de morte (Omran, 1971).

Nos países industrializados, outro aspecto da transição epidemiológica foi observado: idade da ação tardia das doenças crônicas e degenerativas (Oshansky \& Ault, 1986). A característica geral desta fase é expressa pelo declínio das taxas de mortalidade por algumas doenças crônico-degenerativas entre a população adulta de ambos os sexos, retardando o efeito da mortalidade por doenças crônico-degenerativas sem alterar a composição das causas básicas de morte, produzindo, mesmo assim, um aumento na expectativa de vida das populações mais jovens. O conseqüente aumento da extensão da vida nesses países tenderia a provocar o aumento da invalidez e da dependên- cia crescente de serviços sociais e de saúde, por largas parcelas da população.

No presente, o papel das doenças infectocontagiosas na transição epidemiológica passa a ser revisto. Nos países desenvolvidos, observa-se a reemergência dessas doenças, em especial a tuberculose, ligada à AIDS, e resistência aos antibióticos (Smallman-Raynor \& Phillips, 1999; Waters, 2001). No leste europeu, o comportamento reverso das infecto-contagiosas deve-se à desorganização das redes de suporte social e falência do modo de produção vigente nesses países por muitas décadas (Seale, 2000).

Na América Latina, o debate teórico tem enfocado aspectos gerais e regionais da transição epidemiológica na região. No tocante às questões gerais, a transição epidemiológica, de acordo com Frenk et al. (1989a), constitui-se numa das vertentes da "transição da saúde", estando relacionada às condições de saúde. A outra vertente está relacionada à transição dos serviços de saúde, ou seja, à resposta social organizada às mudanças do perfil epidemiológico.

Em relação às questões regionais, Frenk et al. (1989b) sugerem um novo modelo para esta região: modelo tardio-polarizado (protactedpolarised model), devido à não-aderência de países com economias intermediárias, como o Brasil, ao modelo proposto por Omran (1971).

O modelo tardio-polarizado (Frenk et al., 1989a) é assim definido em função da ausência de uma resolução do processo transicional em muitos países, dada a justaposição das patologias infecciosas e crônico-degenerativas ou dupla carga de doenças (Bobadilla \& Possas, 1993).

Outras características destacam-se no modelo proposto por Frenk et al. (1989b): diferenças sociais e regionais na distribuição de bens e serviços de promoção da saúde; fenômeno da contratransição: que é o retorno de doenças já erradicadas ou controladas; seqüência de eras sugeridas por Omran varia em diferentes países e, por fim, grupos sociais distintos apresentam diferentes padrões epidemiológicos.

No tocante à polarização epidemiológica, a magnitude das diferenças sugeririam um padrão assimétrico quanto aos diferenciais na exposição aos fatores de risco e no acesso aos bens e serviços de saúde por parte dos diferentes grupos populacionais (Duarte et al., 2002).

No Brasil, Barreto et al. (1996) têm enfatizado a necessidade da revisão da teoria da transição epidemiológica segundo o contexto específico da América Latina. As principais características das mudanças no padrão epidemiológico no Brasil seriam: (a) permanência de grandes endemias em algumas regiões do país; (b) taxas de mortalidade ainda altas quando comparadas 
com às dos países desenvolvidos; (c) importantes variações geográficas quanto aos padrões epidemiológicos e aos serviços de saúde (Araújo, 1992; Barreto \& Carmo, 1995; Prata, 1992).

A abordagem ecossistema social e saúde é apresentada por Possas (2001) enquanto abordagem teórica alternativa à teoria da transição epidemiológica. Para esta autora, a teoria da transição erraria ao tentar realizar a predição de um provável desenvolvimento dos padrões epidemiológicos apoiado apenas nos estudos sobre os países desenvolvidos nos últimos dois séculos (Possas \& Marques, 1994). A heterogeneidade das sociedades contemporâneas impõe um padrão de risco de tênue fronteiras, a saber, os espaços urbano, rural e selva se interconectam, e riscos e patologias modernas e arcaicas se sobrepõem (Possas, 2001).

A complexidade e a diversidade dos contextos epidemiológicos em vários países apontariam para a necessidade de uma abordagem teórica mais flexível e de conteúdo menos determinista. Também Barreto et al. (1993) sugerem que esta é a principal debilidade do esquema teórico da transição epidemiológica. Acrescem que esta abordagem tenderia a enfatizar a tecnologia médica como principal alternativa interveniente no curso da transição, desconsiderando o papel que as variáveis econômicas e sociais desempenham neste processo (Barreto \& Carmo, 1995; Barreto et al., 1993).

A crítica mais contundente às formulações teóricas sobre a transição da mortalidade vem de Murray \& Chen (1993). Para estes, não há nenhum padrão universal de transição da mortalidade e sim várias transições em curso, muitas das quais em um mesmo país, sejam por questões demográficas (sexo, idade, migrantes), sejam por questões sócio-econômicas (renda, escolaridade).

Entretanto, todos os autores revistos baseiam-se em estudos que analisam populações em altos níveis de agregações espaciais. A dimensão intra-urbana da transição epidemiológica não é observada, isto é, quando analisados desagregadamente, os padrões decorrentes de uma determinada transição epidemiológica revelariam múltiplas transições que estariam em curso em um agregado urbano. Não seriam apenas diferenças no estágio de desenvolvimento da transição em curso. Haveria, ainda, diferenças na forma da transição em si: nas doenças que dominariam o padrão epidemiológico, na seqüência da transição, na composição dos grupos etários afetados pelas causas básicas de morte e, mesmo, diferenças de gênero.

A seguir, o texto apresenta um estudo de indicadores demográficos e epidemiológicos de
Belo Horizonte, tomando enquanto referência os diferenciais intra-urbanos no tocante ao baixo grau de escolarização do chefe do domicílio. Este exercício busca evidenciar que a transição epidemiológica tem múltiplas dimensões que não podem ser plenamente expressas valendose de um único vetor, tampouco com base na observação exclusiva de grandes aglomerados populacionais. As populações urbanas constituem-se em agregados complexos, que apresentam diferentes padrões epidemiológicos e demandas de saúde que precisam ser consideradas quando da confecção das políticas de saúde. Os modelos de transição epidemiológica podem, quando acrescidos da dimensão sócio-econômica e cultural, permitir a aferição da evolução dos padrões epidemiológicos e ocasionar políticas sociais específicas para este tecido social diversificado.

\section{Métodos}

As Unidades de Planejamento (UP) de referência da Secretaria de Planejamento de Belo Horizonte, em número inicial de 81, são as unidades geográficas utilizadas neste estudo. Seis UP foram excluídas do estudo por possuírem áreas com baixa densidade populacional no ano de 1994, além de se constituírem em aglomerados atípicos no interior do tecido urbano. Para estas unidades espaciais excluídas, as estimativas populacionais indicavam populações variando de 28 a 2.218, em 1994. Dessa forma, 75 UP foram consideradas neste estudo. O processo de compatibilização da malha de UP com a dos setores censitários de 1991 e 1996 é detalhada em outras publicações (Paes-Sousa, 2000).

Para o cálculo das taxas de mortalidade e construção das pirâmides etárias, as UP foram agrupadas em quintis, com cada quintil contendo 15 UP. Para a construção das pirâmides etárias presentes na Figura 1, as áreas dos quintis nos dois anos considerados são as mesmas, prevalecendo sempre a condição aferida em 1991. Tanto na Figura 1 quanto nas tabelas subseqüentes, o 1o quintil contém áreas com a menor proporção de chefes de domicílio com baixa escolaridade, vale dizer, as áreas mais afluentes. $\mathrm{O}$ 5o quintil representa o oposto da situação anterior, contendo as áreas com a maior proporção de chefes de domicílio com baixa escolaridade.

As populações das UP para 1994 foram estimadas com base nos dados do Censo de 1991 e da Contagem Inter-Censitária de 1996 (IBGE, 1997). Taxas anuais de crescimento exponencial foram utilizadas para o cálculo das estimativas populacionais (Shryock et al., 1976). 
Figura 1

Estrutura etária de acordo com a proporção de chefes de domicílio com baixa escolaridade.

Belo Horizonte, Minas Gerais, Brasil, 1991 e 1996.

Figura 1a

Primeiro quintil (1991)

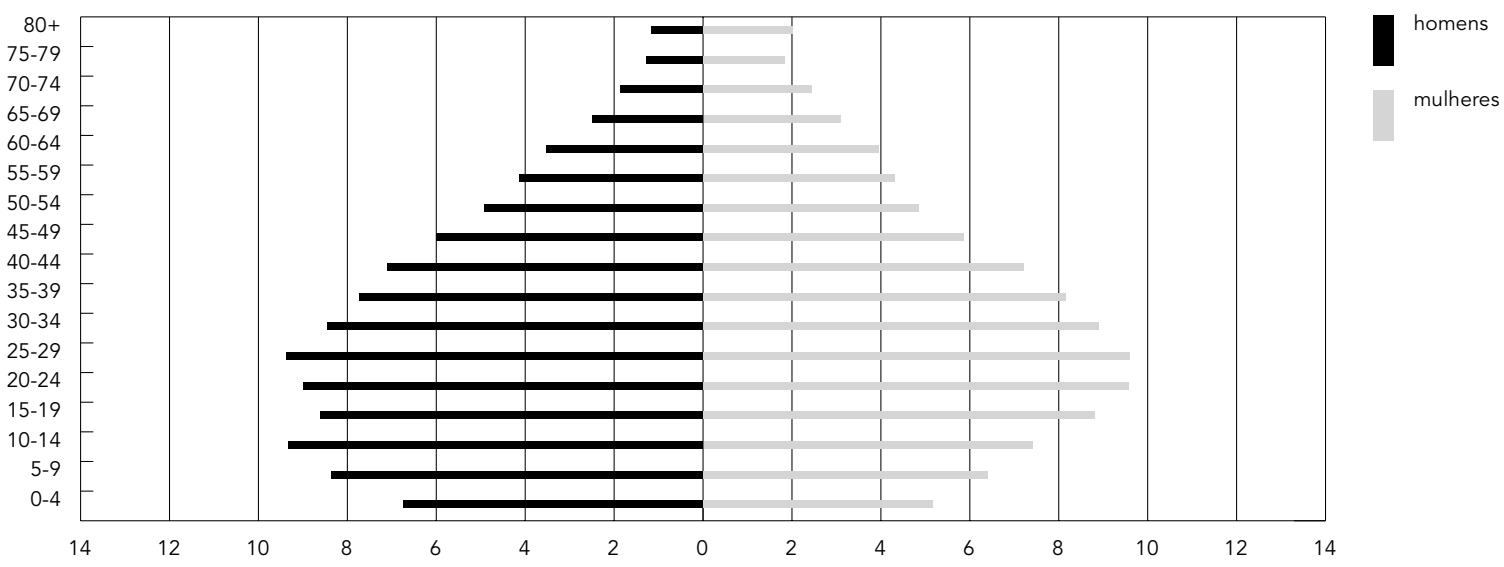

Figura $1 b$

Primeiro quintil (1996).

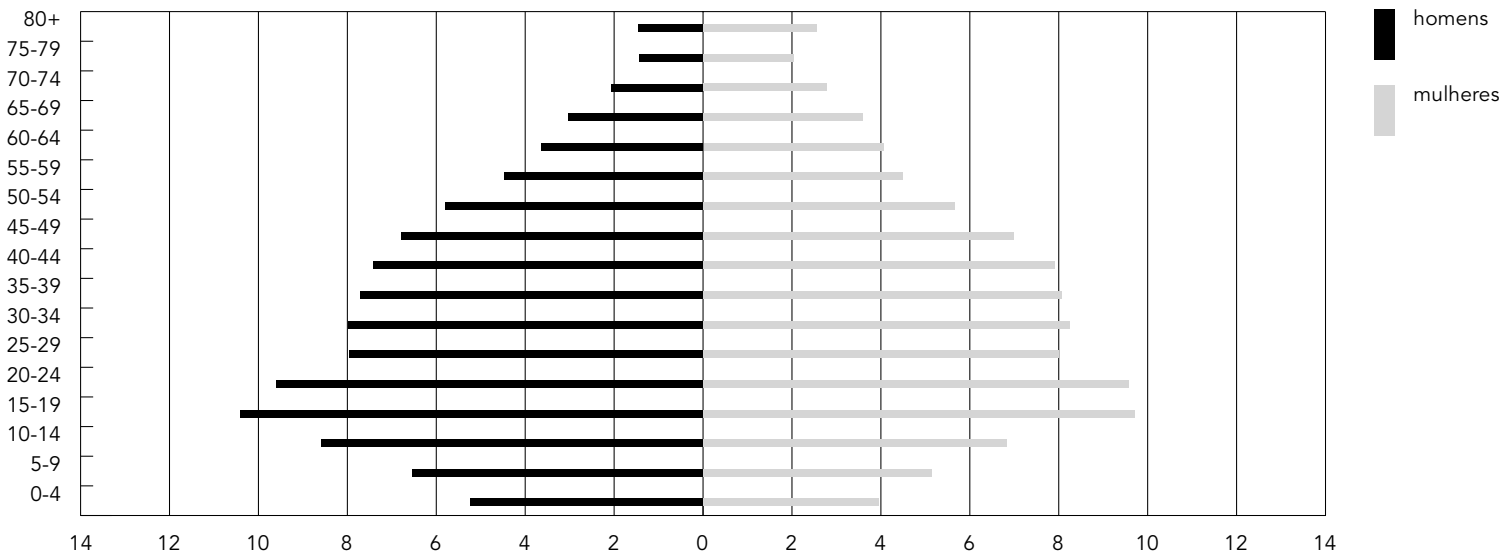

(continua) 
Figura 1 (continuação)

Estrutura etária de acordo com a proporção de chefes de domicílio com baixa escolaridade.

Belo Horizonte, Minas Gerais, Brasil, 1991 e 1996.

Figura 1c

Quinto quintil (1991).

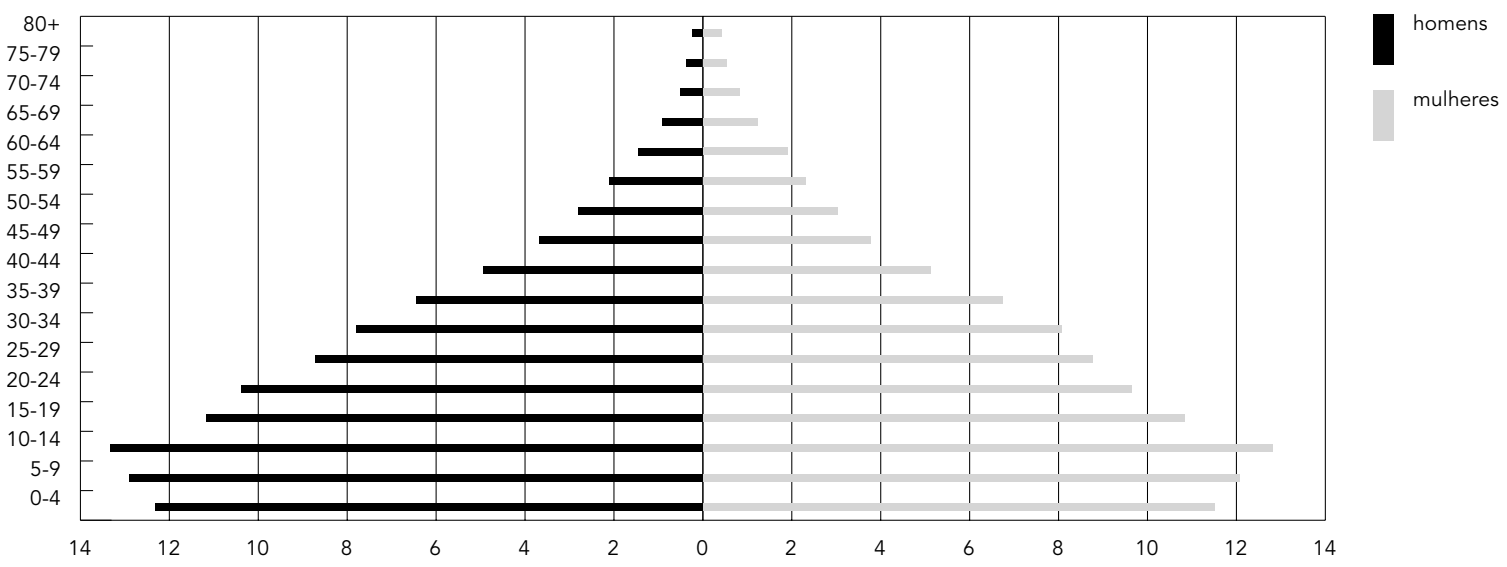

Figura $1 d$

Quinto quintil (1996).

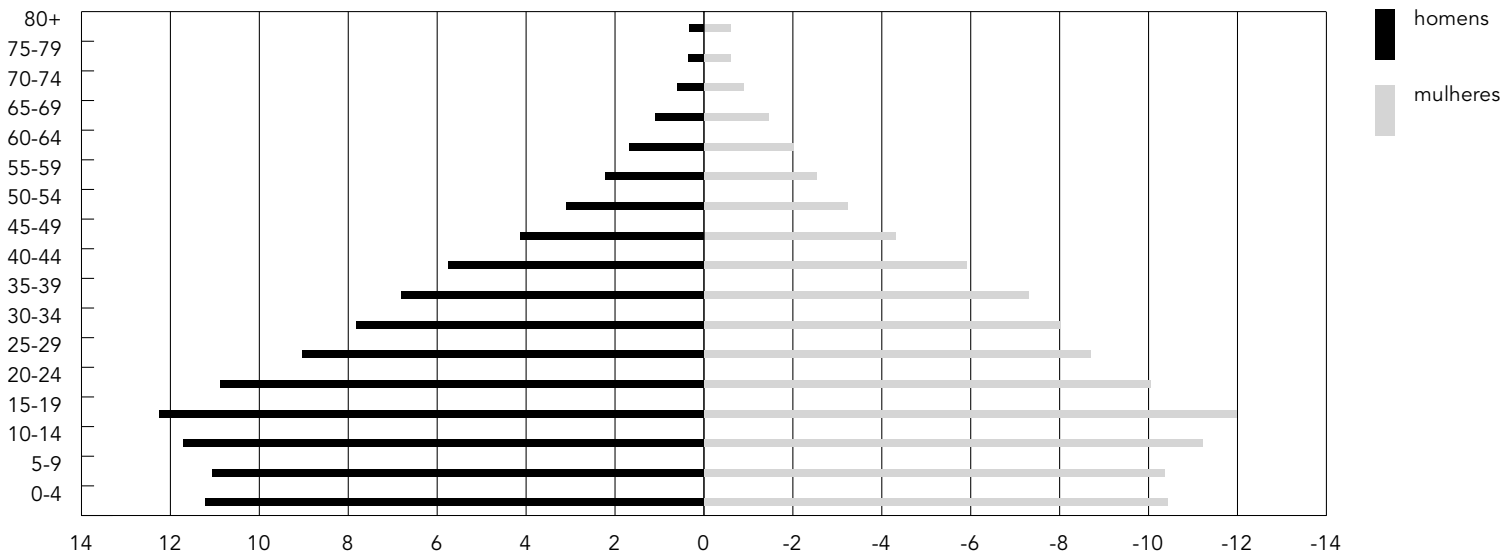


A baixa escolaridade foi medida mediante proporção de chefes de domicílio com até 10 anos de ensino formal em 1991, de acordo com os dados do Censo Populacional de 1991. As proporções encontradas em 1991 foram aplicadas ao ano de 1994.

A revisão das 10.558 Declarações de Óbito (DO) do ano de 1994 compreendeu um período de nove meses. Na primeira etapa do trabalho, procedeu-se à revisão das causas básicas de óbito registradas nas DO dos maiores de 1 ano, pelo Sistema de Informação da Mortalidade (SIM, versão 3.2) (DATASUS, 1998). O SIM, desenvolvido pelo Ministério da Saúde, define de forma automatizada a causa básica do óbito. As DO de menores de um ano já estavam "codificadas” pela Fundação João Pinheiro, que, no momento da coleta de dados desta pesquisa, era o órgão governamental responsável pelas estatísticas vitais do Estado de Minas Gerais.

$\mathrm{Na}$ segunda etapa, foram introduzidos os endereços no Sistema com o auxílio da versão 2.2 do SIM. Esta versão possui um módulo de endereçamento desenvolvido pela Empresa de Informática e Informação de Belo Horizonte (PRODABEL), que não estava disponível na versão seguinte do SIM, no momento em que as DO utilizadas neste estudo foram processadas.

Foram calculadas as taxas padronizadas por idade e sexo de mortalidade geral, mortalidade específica para o grupo etário de 0-4 anos e mortalidade por grupos de causas. Utilizouse a Classificação Internacional de Doenças 9a Revisão (OMS, 1985) no agrupamento das causas básicas de óbito. No cálculo das taxas específicas por causa básica de óbito, os óbitos considerados foram os que apresentaram os seguintes códigos: Doenças Infecciosas (Capítulo I), Doenças do Aparelho Circulatório (Capítulo VII), Doenças do Aparelho Respiratório (Capítulo VIII), Causas Externas de Óbito (Classificação Suplementar E) e Homicídios (E960 E969).

A estratégia adotada, para dar comparabilidade entre as UP, foi a padronização de suas populações por sexo e idade (grupos etários de cinco anos). A população de referência para a padronização foi a população urbana do Estado de Minas Gerais, em 1991.

\section{Resultados: transições demográfica e epidemiológica em Belo Horizonte}

\section{Estruturas etárias}

A Figura 1 mostra as mudanças demográficas nas UP classificadas em quintis de acordo com a baixa escolaridade dos chefes de domicílio em 1991 e em 1996, em Belo Horizonte.

Na Figura 1, as áreas com alta escolaridade (1o quartil) sugerem níveis moderados de mortalidade e queda evidente da fecundidade em 1991. O formato de pêra torna-se mais acentuado em 1996. Neste último ano, o notável crescimento de adultos jovens (15-19, 20-24) pode ser atribuído à imigração direcionada à atividade escolar. Esta hipótese é compatível com o fato de o Município possuir uma das maiores concentrações de instituições de ensino superior no Brasil. No entanto, tal fato não explica porque este fenômeno não está presente em 1991. É possível especular-se que os diferenciais estejam ligados a mudanças metodológicas na coleta de dados em relação à definição do chefe do domicílio ou a alguma variação temporal na demanda por escolaridade superior devido às motivações econômicas e/ou culturais.

As pirâmides representando áreas com baixa escolaridade (5o quintil) sugerem alta fecundidade e moderada mortalidade em 1991. O estreitamento da base da pirâmide em 1996 indica que uma etapa mais acentuada da transição da fecundidade pode estar se iniciando. A permanência de uma base larga na pirâmide pode estar relacionada ao "momentum" demográfico, quando uma grande quantidade de mulheres em idade fértil gera, no conjunto, um número expressivo de crianças apesar das baixas taxas de fecundidade. Entretanto, a situação pode ainda indicar uma atitude menos determinada de controle da fecundidade em função das incertezas da sobrevivência dos infantes.

\section{Mortalidade proporcional}

A Figura 2 apresenta a distribuição relativa de mortalidade por grupo de causa no Município de Belo Horizonte, de 1900 até 1995. Os dados referentes aos anos de 1990 e 1995 foram extraídos do Departamento de Informática do SUS (DATASUS, 1998). Os dados dos demais anos foram extraídos de Maletta (1997).

Para efeito deste debate, é importante que se destaque na Figura 2 que: de 1950 em diante, as doenças do aparelho circulatório assumem a liderança dentre as causas básicas de óbito; desde 1960, as causas externas e as neoplasias aparecem enquanto importantes causas de morte e as doenças infeciosas e, possivelmente, causas perinatais estão em franco declínio.

A Figura 2 apresenta um clara tendência: Belo Horizonte caminha em marcha acelerada na direção de um padrão moderno de mortalidade. Todavia, o que está escondido no interior desta 
assertiva é que os ganhos, o ritmo e o mecanismo destas transições variam de acordo com as condições sócio-econômicas das populações.

\section{Mortalidade intra-urbana}

A Tabela 1 mostra taxas brutas e taxas padronizadas de mortalidade por idade e sexo de acordo com a proporção de chefes de domicílio com baixa escolaridade em 75 UP em Belo Horizonte. A taxas padronizadas apresentam elevação inversa à escolaridade dos chefes de domicílio. As áreas com dominância de chefes com baixa escolaridade (5o quintil) apresentam taxas padronizadas $56 \%$ maiores que as das áreas afluentes (1ㅇquintil).

Figura 2

Distribuição relativa da mortalidade por grupo de causa.

Belo Horizonte, Minas Gerais, Brasil, 1900-1995.
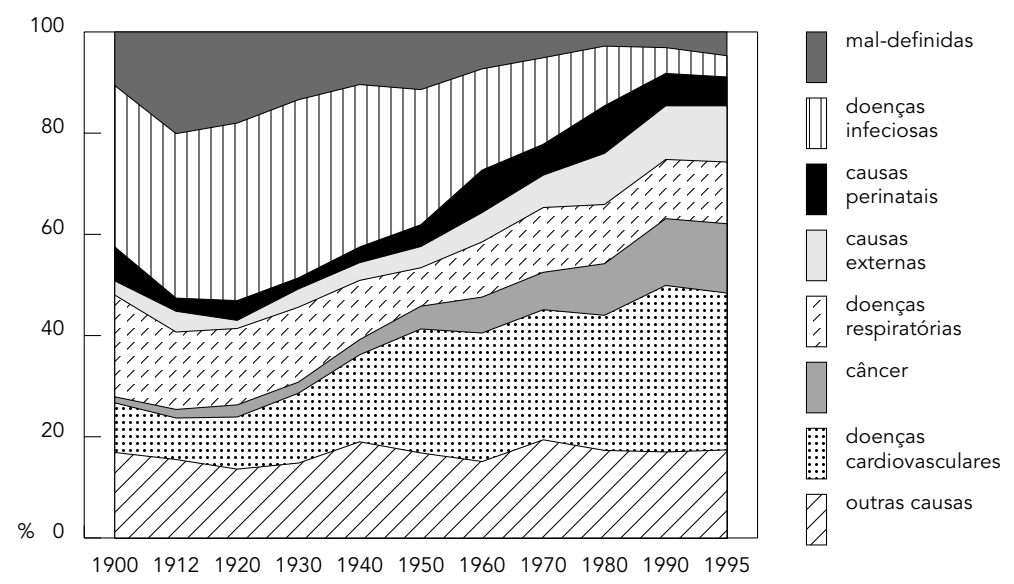

Tabela 1

População, óbitos, taxas de mortalidade geral (brutas e padronizadas por sexo e idade) de acordo com a proporção de chefes de domicílio de baixa escolaridade (/10.000) em Belo Horizonte, Minas Gerais, Brasil, 1994.

\begin{tabular}{|c|c|c|c|c|c|c|}
\hline & \multicolumn{5}{|c|}{ Chefes de domicílio com baixa escolaridade - quintis } & \multirow[t]{2}{*}{ Total } \\
\hline & $\begin{array}{l}\text { 1ㅇ (alta } \\
\text { escolaridade) }\end{array}$ & 2으 & 3으 & 4으 & $\begin{array}{l}\text { 5ㅇ (baixa } \\
\text { escolaridade) }\end{array}$ & \\
\hline População & 327.887 & 501.068 & 450.878 & 455.200 & 317.906 & 2.052 .939 \\
\hline Óbitos & 1.735 & 2.813 & 2.352 & 2.212 & 1.446 & 10.558 \\
\hline Taxas brutas & 52,91 & 56,14 & 52,16 & 48,59 & 45,49 & 51,43 \\
\hline Taxas padronizadas & 37,27 & 50,37 & 55,08 & 57,29 & 58,01 & 50,99 \\
\hline
\end{tabular}

A Figura 3 mostra que o padrão acima descrito é mais acentuado no grupo etário de 0-4 anos. Nas áreas de alta escolaridade dos chefes de domicílio (1o quintil), o valor da taxa de mortalidade padronizada por sexo é quase a metade dos valores encontrados nas áreas de escolaridade mais baixa (5o quintil).

\section{Taxas específicas de mortalidade por causa básica de óbito}

A Tabela 2 apresenta taxas de mortalidade específicas padronizadas por sexo e idade de acordo com as causas básicas de óbito selecionadas. Elas enfocam as seguintes causas básicas de óbitos: doenças do aparelho circulatório, doenças do aparelho respiratório, doenças infecciosas, causas externas e homicídios. Em geral, as populações das áreas, cujos chefes de domicílio têm menor escolaridade, apresentam taxas mais elevadas. As taxas indicam que os padrões de mortalidade variam inversamente, de acordo com a escolaridade do chefe do domicílio.

Há um hiato entre o 1o (alta escolaridade) e 5 o quintil (baixa escolaridade), indicando um padrão desigual de mortalidade. Em comparação com o 1 quintil, o 5o quintil apresenta taxas de mortalidade mais altas para todos os grupos de causa selecionados, quais sejam: 2,7 vezes maior (doenças infecciosas), 1,8 vezes (aparelho circulatório), 1,7 vezes (aparelho respiratório), 2,7 vezes (infecciosas), 1,9 vezes (causas externas), 4,1 maior (homicídios).

\section{Conclusão}

A apresentação de dados agregados em nível municipal não revela a polarização intra-urbana existente. O estudo intra-urbano dos padrões demográficos e epidemiológicos pode 
contribuir com a formulação de políticas e planejamento das ações de saúde com observações mais precisas das necessidades de saúde das populações.

Os dados de Belo horizonte sugerem que os padrões demográficos e epidemiológicos variam de acordo com o status sócio-econômico da UP. Em 1991, as pirâmides populacionais que representam as áreas com maior proporção de chefes de domicílio com baixa escolaridade indicavam a persistência de níveis elevados de fertilidade associados à moderada mortalidade. Já em 1996, essas mesmas áreas apresentavam uma base da pirâmide ligeiramente mais estreitada, sugerindo que o processo de transição da fecundidade estava se iniciando.

Os achados suportam a hipótese de que as grandes cidades brasileiras produzem um padrão complexo e diversificado de mortalidade. Essa cidades experimentam um padrão em um processo de transição múltipla de mortalidade no qual as doenças infecciosas passam a ser substituídas pelas causas externas como um dos principais grupos de causa de morte nas áreas mais pobres.

Os grupos mais ricos exibem um padrão avançado de mortalidade dominado por doenças cardiovasculares e neoplasias. As áreas mais pobres vivenciam a carga dupla de doenças, compreendendo populações mais vulneráveis à mortalidade por doenças infecciosas, notadamente os menores de 5 anos, homicídios, doenças cardiovasculares e doenças respiratórias. Este padrão múltiplo pode impactar a estrutura demográfica das populações mais pobres de duas formas: produzindo um excesso de óbitos precoces e interferindo nos níveis de fecundidade.

Se as principais contribuições dos modelos de transição demográfica e epidemiológica são a antevisão de cenários a serem vividos pelas populações objetivando a elaboração de políticas específicas, a redução do estudo a uma observação de dados agregados implicará o desenvolvimento de "políticas de padrão único", que tendem a não satisfazer a nenhum dos grupos sociais residentes nos centros urbanos.

Se as cidades não são aglomerados humanos homogêneos no tocante à dimensão sócioeconômica e tampouco aos perfis demográfico-epidemiológicos, não é adequado que se disponibilizem modalidades de serviços de saúde tendo-se como referência apenas o tamanho da população. Populações distintas, quanto às

Figura 3

Taxa de mortalidade geral padronizada por sexo (0-4 anos) segundo baixa escolaridade do chefe do domicílio (quintis). Belo Horizonte, Minas Gerais, Brasil, 1994.

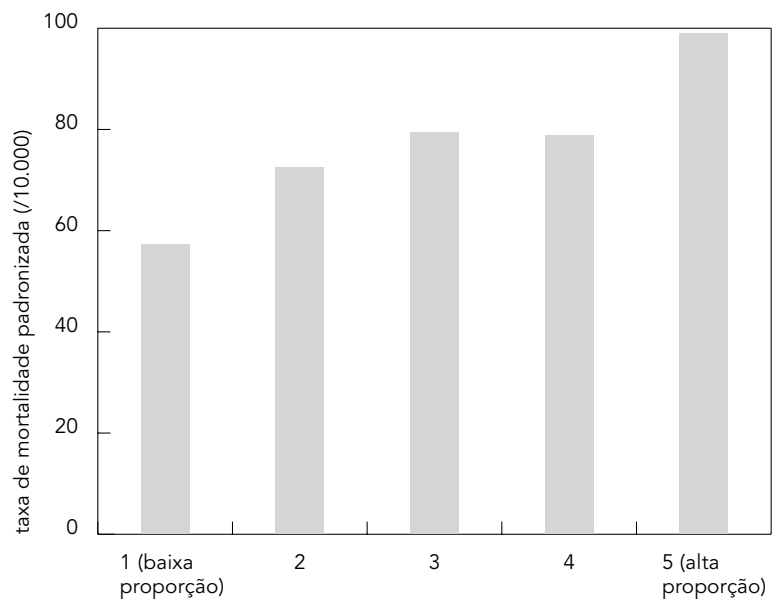

Tabela 2

Óbitos, taxas de mortalidade padronizadas por sexo e idade (/10.000) segundo escolaridade do chefe do domicílio (quintis). Belo Horizonte, Minas Gerais, Brasil, 1994.

\begin{tabular}{|c|c|c|c|c|c|c|c|c|c|c|}
\hline \multirow{2}{*}{$\begin{array}{l}\text { Quintil } \\
\text { (educação) }\end{array}$} & \multicolumn{2}{|c|}{ Circulatório } & \multicolumn{2}{|c|}{ Respiratório } & \multicolumn{2}{|c|}{ Infecciosas } & \multicolumn{2}{|c|}{ Causas externas } & \multicolumn{2}{|c|}{ Homicídios } \\
\hline & óbitos & taxas & óbitos & taxas & óbitos & taxas & óbitos & taxas & óbitos & taxas \\
\hline Total & 3.395 & 15,86 & 1.348 & 6,65 & 520 & 2,65 & 970 & 4,61 & 232 & 1,09 \\
\hline 1 (alta) & 605 & 10,96 & 225 & 4,35 & 48 & 1,38 & 107 & 2,93 & 17 & 0,51 \\
\hline 2 & 949 & 15,75 & 396 & 7,17 & 114 & 2,28 & 214 & 3,97 & 37 & 0,70 \\
\hline 3 & 749 & 17,55 & 301 & 7,30 & 123 & 2,90 & 212 & 4,63 & 53 & 1,11 \\
\hline 4 & 675 & 18,73 & 253 & 6,98 & 126 & 3,17 & 271 & 6,01 & 56 & 1,20 \\
\hline 5 (baixa) & 417 & 19,69 & 173 & 7,29 & 109 & 3,85 & 166 & 5,50 & 69 & 2,09 \\
\hline
\end{tabular}


suas necessidades de saúde, demandam serviços de saúde distintos.

A não-observância das necessidades de saúde das populações resulta na insuficiência de serviços de saúde associada ao desperdício de recursos, vale dizer, alocação inadequada de recursos.

A melhoria dos bancos de dados associado aos avanços tecnológicos no processamento das informações, especialmente as tecnologias de geoprocessamento, permitem uma melhor aferição das necessidades de saúde das populações urbanas.

\section{Agradecimentos}

Este artigo contém parte dos achados da tese de doutoramento do autor na London School of Hygiene and Tropical Medicine (University of London). Este programa de doutoramento foi financiado pela Fundação de Coordenação de Aperfeiçoamento de Pessoal de Nível Superior (CAPES).

\section{Referências}

ARAÚJO, J., 1992. Polarização epidemiológica no Brasil. Informe Epidemiológico do SUS, 1:5-16.

BARRETO, M. L. \& CARMO, E., 1995. Mudanças em padrões de morbi-mortalidade: Conceitos e métodos. In: Velhos e Novos Males da Saúde no Brasil: A Evolução do País e suas Doenças (C. Monteiro, org.), pp. 17-30, São Paulo: Editora Hucitec/ Núcleo de Pesquisas Epidemiológicas em Nutrição e Saúde, Universidade de São Paulo.

BARRETO, M. L.; CARMO, E. H.; NORONHA, C. V.; NEVES, R. B. T. \& ALVES, P. C., 1993. Mudança nos padrões de morbi-mortalidade: Uma revisão crítica das abordagens epidemiológicas. Physis, 3 : 126-146

BARRETO, M. L.; CARMO, E.; SANTOS, C. E. \& FERREIRA, L., 1996. "Emergentes", "re-emergentes" e "permanecentes": Tendências recentes das doenças infecciosas e parasitárias no Brasil. Informe Epidemiológico do SUS, 3:7-17.

BEMFAM (Sociedade Civil Bem-Estar Familiar no Brasil), 1997. Brasil, Pesquisa Nacional sobre Demografia da Saúde - 1996. Rio de Janeiro: BEMFAM

BOBADILLA, J. L. \& POSSAS, C. A., 1993. Health policy issues in three Latin American Countries: Implications of the epidemiological transition. In: The Epidemiological Transition: Policy and Planning Implications for Developing Countries (J. N. Gribble \& S. H. Preston, ed.), pp. 145-169, Washington, DC: National Academy Press.
CHESNAIS, J. C., 1992. The Demographic: TransitionStages, Patterns, and Economic Implications. New York: Claredon Press/Oxford University Press.

DATASUS (Departamento de Informática do Sistema Único de Saúde), 1998. Sistema de Informações sobre Mortalidade; 1979 - 1997: Dados de Declaração de Óbito. CD-ROM. Brasília: Ministério da Saúde.

DUARTE, E.; SCHINEIDER, M. C.; PAES-SOUSA, R.; RAMALHO, W. M.; SARDINHA, L.; BARBOSA-SILVA, J. \& CASTILLO-SALGADO, C., 2002. Epidemiologia das Desigualdades no Brasil: Um Estudo Exploratório. Brasília: Organização Pan-Americana de Saúde.

FRENK, J.; BOBADILLA, J. L.; SEPULVEDA, J. \& LOPEZ-CERVANTES, M., 1989a. Health transition in middle-income countries: New challenges for health care. Health Policy and Planning, 4:29-39.

FRENK, J.; FREJKA, T.; BOBADILLA, J. L.; STERN, C.; SEPULVEDA, J. \& JOSE, M., 1989b. The epidemiological transition in Latin America. In: International Population Conference, Proceedings, pp. 419-431, Liège: The International Union for the Scientific Study of Population.

IBGE (Fundação Instituto Brasileiro de Geografia e Estatística), 1997. Contagem de População 1996. Rio de Janeiro. Rio de Janeiro: IBGE.

IBGE (Fundação Instituto Brasileiro de Geografia e Estatística), 2001. Censo Demográfico 2000, Sinopse Preliminar. 2 Maio $2001<$ http:/ / www.ibge. 
gov.br/ibge/estatistica/populacao/censo2000/ default.shtm>.

MALETTA, C. H. M., 1997. A Cidade e os Cidadãos: Belo Horizonte - 100 anos. Belo Horizonte: Sografe Editora.

MARTINE, G., 1993. A Redistribuição Espacial da População Brasileira Durante a Década de 80. Brasília: Instituto de Pesquisas Econômicas e Aplicadas/Instituto Sociedade População e Natureza.

MARTINE, G., 1994. Estado, economia e mobilidade geográfica: Retrospectiva e perspectivas para o fim do século. Revista Brasileira de Estudos de População, 11:41-60.

MARTINE, G., 1996. Brazil's fertility decline, 1965-95: A fresh look at key factors. Population and Development Review, 22:47-75

MURRAY, C. \& CHEN, L., 1993. In search for a contemporary theory for understanding mortality change. Social Science and Medicine, 36:143-155.

OLSHANSKY, J. \& AULT, B., 1986. The fourth stage of the epidemiologic transition: The age of delayed degenerative diseases. Milbank Memorial Fund Quarterly, 64:355-391.

OMRAN, A. R., 1971. The epidemiologic transition: A theory of the epidemiology of population change. Milbank Memorial Fund Quarterly, 49:509-358.

OMS (Organização Mundial da Saúde), 1985. Manual da Classificação Estatística Internacional de Doenças, Lesões e Causas de Óbitos - Nona Conferência de Revisão. São Paulo: Centro Brasileiro de Classificação de Doenças em Português.

PAES-SOUSA, R., 2000. Socioeconomic and Environmental Differentials, and Mortality in a Developing Urban Area (Belo Horizonte - Brazil). Ph.D. Thesis, London: London School of Hygiene and Tropical Medicine, University of London.
PATARRA, N., 1994. Transição demográfica: Novas evidências, velhos desafios. Revista Brasileria de Estudos de População, 11:27-40.

PHILIPS, D., 1994. Does epidemiological transition have utility for health planners? Social Science and Medicine, 38:vii- $\mathrm{x}$.

POSSAS, C. A \& MARQUES, M. B., 1994. Health transitions and complex systems: A challenge to prediction? Annals of New York Academy of Science, 740:85-96.

POSSAS, C., 2001. Social ecosystem health: Confronting the complexity and emergence of infcctious diseases. Cadernos de Saúde Pública, 17:31-41.

PRATA, P., 1992. A transição epidemiológica no Brasil. Cadernos de Saúde Pública, 8:168-175.

SEALE, C., 2000. Changing patterns of death and dying. Social Science and Medicine, 51:917-930.

SHRYOCK, H., SIEGEL, J. \& STOCKWELL, E., 1976. The Methods and Materials of Demography. San Diego: Academic Press.

SKELDON, R., 1990. Population Morbility in Developing Countries. London: Behaven Press.

SMALLMAN-RAYNOR, M. \& PHILLIPS, D., 1999. Late stages of epidemiological transition: Health status in developed world. Health Place, 5:209-222.

WATERS, W. F., 2001. Globalization, socioeconomic restructuring, and community health. Journal of Community Health, 26:79-92.

Recebido em 30 de maio de 2001

Versão final reapresentada em 12 de dezembro de 2001

Aprovado em 3 de abril de 2002 